\title{
Intracellular iodinated compounds affect sodium iodide symporter expression through TSH-mediated signaling pathways
}

\author{
HUIBIN HUANG ${ }^{1}$, YAXIONG SHI ${ }^{1}$, LING LIN ${ }^{2}$, XISHENG LI $^{1}$, \\ LIANGYI LI ${ }^{1}$, XIAHONG LIN ${ }^{1}$ and DONGMING XU ${ }^{2}$ \\ Divisions of ${ }^{1}$ Endocrinology, and ${ }^{2}$ Rheumatism, Second Affiliated Hospital of Fujian Medical University, Fujian, P.R. China
}

Received July 30, 2010; Accepted October 15, 2010

DOI: $10.3892 / \mathrm{mmr} .2010 .384$

\begin{abstract}
The mechanisms by which thyroid stimulating hormone (TSH) regulates the expression and activity of sodium iodide symporter (NIS) through cAMP-PKA have been partially elucidated by many studies. However, the effects of the TSH-mediated PLC-IP3 signaling pathway on the expression of NIS and how intracellular iodinated compounds interfere with these signaling pathways are poorly understood. In this study, we investigated the effects of the TSH-mediated cAMP-PKA and PLC-IP3 pathways on the expression of NIS in the presence of various intracellular iodinated compounds. The intracellular iodinated compounds were formed by treating cells with different concentrations of iodine with or without methimazole (MMI), an inhibitor of iodine organification, in a pig monolayer thyrocyte in vitro. A high concentration of iodine increased NIS expression at the mRNA and protein levels; however, this phenomenon was not observed in the presence of MMI. Both the cAMP-PKA and PLC-IP3 signaling pathways inhibited the expression of NIS at low iodine concentrations; however, in thyrocytes treated with high concentrations of iodine, the effect of cAMP-PKA on the expression of NIS changed from inhibition to promotion, while the PLC-IP3 pathway continued to inhibit NIS expression. These findings indicate that intracellular iodinated compounds affect NIS expression through the TSH-mediated cAMP-PKA and PLC-IP3 pathways.
\end{abstract}

\section{Introduction}

Sodium iodide symporter (NIS), a membrane glycoprotein localized at the basolateral plasma membrane of thyrocytes (1), plays an essential role in the accumulation of iodine in the thyroid. Iodine accumulation is a key step in thyroid hormone synthesis and is crucial in the diagnosis and treatment of

Correspondence to: Dr Ling Lin, Division of Rheumatism, Second Affiliated Hospital of Fujian Medical University, Fujian, P.R. China E-mail: dhh7397007@yahoo.com.cn

Key words: iodine, iodinated compounds, sodium iodide symporter, $\mathrm{TSH}$, signal pathway thyroid disorders (2). The expression and activity of NIS are mainly regulated by thyroid stimulating hormone (TSH), iodine $(3,4)$ and thyroglobulin $(\mathrm{Tg})$.

TSH, a major regulator of NIS expression and iodine uptake (5), also induces the activity of NIS protein $(6,7)$ through posttranslational modifications. TSH increases NIS expression and iodine uptake by positively regulating NIS expression at the protein and mRNA levels through the cAMP-PKA-dependent and -independent pathways (8-10). The withdrawal of TSH results in a decrease in intracellular cAMP concentration and iodine uptake activity (11). TSH not only regulates NIS transcription and biosynthesis, but also mediates its activity through a post-transcriptional mechanism essential for NIS trafficking to the membrane in vivo and in vitro (6).

Iodine is another important factor regulating thyroid function. In 1948, Wolff and Chaikoff found that high doses of iodine blocked iodine organification in rat thyroid in vivo. This phenomenon was later called the Wolff-Chaikoff effect. The function regulated by a high dose of iodine is transient; the thyroid adapts to a sustained high dose of iodine, avoiding the effects of iodine overload and resuming organification (12). The Wolff-Chaikoff effect constitutes a highly specialized intrinsic autoregulatory system that protects the thyroid from high doses of iodine and ensures adequate iodine uptake for hormone biosynthesis (13). The mechanisms underlying the Wolff-Chaikoff effect are complex, and involve a reduction in the expression of NIS at the mRNA and protein levels $(12,14)$.

Methimazole (MMI) is an inhibitor of TPO activity; it inhibits iodine organification and abolishes the iodide uptakesuppressing effect under high doses of iodine (15). This phenomenon shows that the Wolff-Chaikoff effect may be mediated by intracellular or follicular iodinated compounds, such as iodine lipids, which have been confirmed to inhibit TSH-mediated adenylate cyclase activity $(16,17)$.

$\mathrm{TSHr}$ is a G protein-coupled receptor that transfers extracellular signals mainly by the TSH/TSHr-cAMP-PKA and TSH/TSHr-PLC-IP3 pathways $(18,19)$. The mechanism by which TSH regulates the expression and activity of NIS through the cAMP-PKA pathway has been partially clarified by several studies. However, the effect of the TSH-mediated PLC-IP3 signaling pathway on the expression of NIS and and how intracellular iodinated compounds interfere with these signaling pathways are not fully understood. 
A

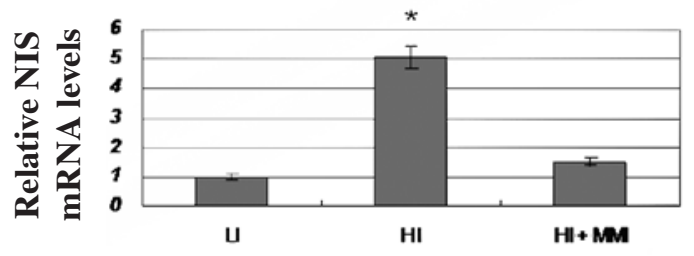

B

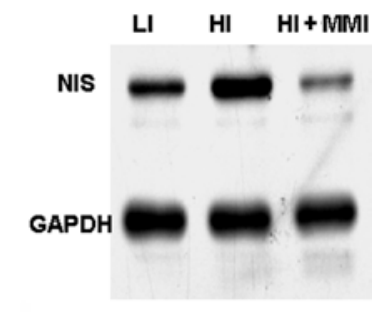

C

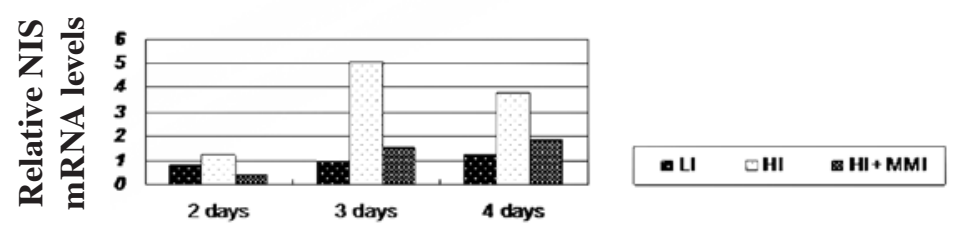

Figure 1. Effects of intracellular iodinated compound on NIS. Pig thyroid primary cells were seeded at a density of $0.2 \times 10^{6}$ cells $/ \mathrm{ml}$ and cultured for 3 days, with TSH added after the cells were seeded for $24 \mathrm{~h}$, to form monolayer cells. The monolayer cells were treated with $0.1 \mu \mathrm{mol} / 1 \mathrm{NaI}$ (LI group), $25 \mu \mathrm{mol} / 1 \mathrm{NaI}$ (HI group) or $25 \mu \mathrm{mol} / 1 \mathrm{NaI}+2 \mathrm{mmol} / 1 \mathrm{MMI}$ (HI + MMI group) for $72 \mathrm{~h}$, respectively. Real-time quantitative RT-PCR and Western blotting were performed to measure changes in (A) NIS mRNA and (B) NIS protein levels (normalized to GAPDH). (C) Time-dependent changes in NIS mRNA ( $\mathrm{n}=3$ ). Results are expressed as the mean \pm SEM. ${ }^{*} \mathrm{P}<0.05$ compared to LI and HI + MMI.

In this study, we investigated the effect of intracellular iodinated compounds on the regulation of NIS expression and the effects of TSH on NIS expression through the cAMP-PKA and PLC-IP3 pathways in the presence of various intracellular iodinated compounds at different concentrations of iodine, with or without MMI, in a pig monolayer thyrocyte in vitro. The aim was to elucidate the effects of different TSH-mediated signaling pathways and intracellular iodinated compounds on the expression of NIS.

\section{Materials and methods}

Thyroid monolayer cell culture. The thyroid was removed from a pig within $2 \mathrm{~h}$ of its being sacrificed, washed several times with sterilized PBS, and then cut into pieces at a volume of $1 \mathrm{~mm}^{3}$ under aseptic conditions. Thyroid fragments were digested with $0.125 \%$ trypsin (Sigma) for $30 \mathrm{~min}$ at room temperature and dispersed into a cell suspension. The suspension was centrifuged at $1,000 \mathrm{rpm}$ for $5 \mathrm{~min}$ after being filtered with a 200 mesh filter. The cells were seeded at a density of $0.2 \times 10^{6}$ cells $/ \mathrm{ml}$ in DMEM medium (Hyclone) containing 10\% bovine serum (Hyclone), $200 \mathrm{IU} / \mathrm{ml}$ penicillin (Sigma) and $200 \mathrm{IU} / \mathrm{ml}$ streptomycin (Sigma), and maintained at $37^{\circ} \mathrm{C}$ with $5 \% \mathrm{CO}_{2}$. TSH $(1 \mathrm{mIU} / \mathrm{ml})($ Merck) was added to the culture medium after the cells were seeded for $24 \mathrm{~h}$.

Administration of iodine and MMI. After TSH treatment and three days of culture, monolayer cells were formed. To investigate the effect of the intracellular iodinated compounds, the monolayers were divided into the high iodine (HI), low iodine (LI) group and high iodine + MMI (HM) groups, treated with $25 \mu \mathrm{mol} / 1$ sodium iodide (Sigma), $0.1 \mu \mathrm{mol} / 1$ sodium iodine and $25 \mu \mathrm{mol} / 1$ sodium iodide (Sigma), and $2 \mathrm{mmol} / \mathrm{l} \mathrm{MMI}$ (Sigma), respectively.

Administration of H89 and LY294002. H89 and LY294002, blockers of PKA and IP3 activity, are commonly used to study the effects of signaling pathways. To investigate the effects of the TSH-mediated cAMP-PKA and PLC-IP3 pathways, the HI and LI groups were treated with H89 and LY294002 at a final concentration of $30 \mu \mathrm{mol} / 1$.

Western blot analysis. The cell lysates were centrifuged at $12,000 \mathrm{~g}$ for $30 \mathrm{~min}$. Protein concentration was determined by the BCA (Sigma) assay. Cell lysates were then electrophoretically separated on $12 \%$ polyacrylamide gels and transferred onto PVDF membranes (Millipore). The membranes were blocked for $1.5 \mathrm{~h}$ at room temperature in $5 \%$ non-fat milk and incubated overnight at $4^{\circ} \mathrm{C}$ with NIS antibodies (Beijing Biosynthesis Biotechnology Co., Ltd.). The membranes were washed three times in TBST for $30 \mathrm{~min}$, incubated with horseradish peroxidase-conjugated secondary antibodies for $45 \mathrm{~min}$, and washed again three times in TBST. Immunoreactive bands were revealed using an enhanced chemiluminescence detection system. The negative group used $2 \%$ BSA instead of the primary antibodies. GAPDH (Beijing Biosynthesis Biotechnology Co., Ltd.) was also detected as an internal control. The X-ray film was scanned and the band density was calculated using ImageJ software (20).

Preparation of total RNA and fluorescent quantitative realtime RT-PCR. Total RNA was isolated from the cells using the RNAiso Plus kit (TaKaRa). RNA precipitate was then dissolved in 10-15 $\mu$ l of RNase-free water and analyzed quantitatively and qualitatively using a spectrophotometer. The integrity of total RNA was determined by $1 \%$ formaldehyde agarose gel electrophoresis. A two-step reverse transcription-polymerase chain reaction (RT-PCR) procedure was performed using the PrimeScript ${ }^{\mathrm{TM}}$ RT reagent kit (TaKaRa) following the manufacturer's instructions. The resulting cDNA was used in the real-time PCR. For PCR amplification, $2 \mu \mathrm{l}$ of the cDNA was used in a $20-\mu l$ reaction mixture. The PCR primers of NIS were 5'-AGTGATGCTGACGGTTTCTGGGTT-3' and 5'-AGGTTGATCCGGAGTGGTTCTT-3'. Hot-start real-time 
A

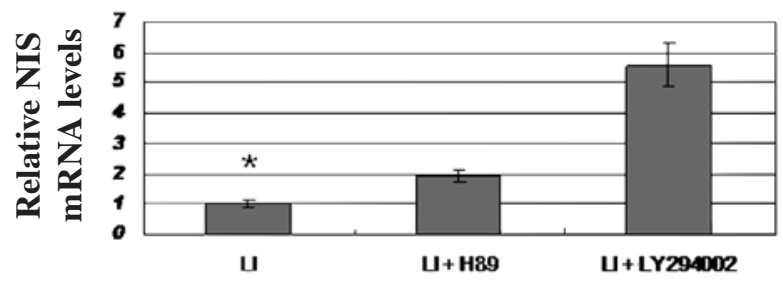

B

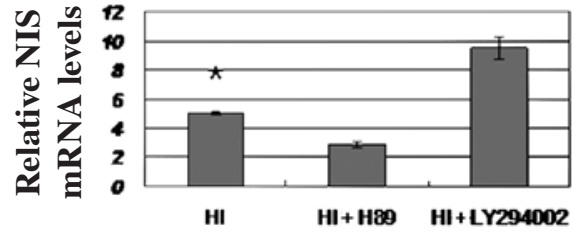

Figure 2. TSH-mediated cAMP-PKA and PLC-IP3 pathways regulate the expression of NIS. Pig thyroid primary cells were seeded at a density of $0.2 \times 10^{6}$ cells $/ \mathrm{ml}$ and cultured for 3 days, with TSH added after the cells were seeded for $24 \mathrm{~h}$, to form monolayer cells. The monolayer cells were then treated with a PKA blocker (H89) or an IP3 blocker (LY294002) for $72 \mathrm{~h}$ under low or high iodine conditions. Real-time quantitative RT-PCR was performed to measure changes in NIS mRNA (normalized to GAPDH). (A) Changes in NIS mRNA in cells treated with $0.1 \mu \mathrm{mol} / 1 \mathrm{NaI}$ and $30 \mu \mathrm{mol} / /$ H89 (LI + H89 group), or with $0.1 \mu \mathrm{mol} / 1 \mathrm{NaI}$ and $30 \mu \mathrm{mol} / 1 \mathrm{LY} 294002$ $\left(\mathrm{LI}+\mathrm{LY}\right.$ group) $(\mathrm{n}=3)$. Results are expressed as the mean \pm SEM. ${ }^{*} \mathrm{P}<0.05$ compared to the LI + H89 and LI + LY294002 groups. (B) Changes in NIS mRNA in cells treated with $25 \mu \mathrm{mol} / 1 \mathrm{NaI}$ and $30 \mu \mathrm{mol} / 1 \mathrm{H} 89$ (HI + H89 group), or with $25 \mu \mathrm{mol} / 1 \mathrm{NaI}$ and $30 \mu \mathrm{mol} / 1 \mathrm{LY} 294002$ (HI + LY294002 group) $(\mathrm{n}=3)$. Results are expressed as the mean $\pm \mathrm{SEM}$. ${ }^{*} \mathrm{P}<0.05$ compared to the HI + H89 and HI + LY294002 groups.

PCR was performed using SYBR green-based detection in a Rotor-Gene 6000 , with initiation at $95^{\circ} \mathrm{C}$ for $30 \mathrm{sec}$, then 40 cycles of a two-step PCR comprising 5 sec of denaturation at $95^{\circ} \mathrm{C}$ and $30 \mathrm{sec}$ of annealing/elongation at $60^{\circ} \mathrm{C}$. GAPDH was amplified as an internal control using the PCR primers 5'-GAAGGTCGGAGTGAACGGAT-3' and 5'-CATGGGTAGAATCATACTGGAACA-3'. Data were analyzed using the appropriate expression software.

Statistical analysis. Statistical differences were determined using the Student's t-test. A p-value of $<0.05$ was considered significant.

\section{Results}

Intracellular iodinated compound regulates the expression of NIS. The expression of NIS significantly increased in the HI group compared to the LI group; the effect was abolished when MMI was administered in the HI group (Fig. 1). These results indicate that intracellular iodinated compounds are responsible for the increase in NIS.

TSH-mediated CAMP-PKA and PLC-IP3 signaling pathways regulate the expression of NIS in the presence of various iodine concentrations. When thyrocytes were treated with H89 and LY294002 separately with low concentrations of iodine, the expression of NIS was significantly increased in both the H89 and LY294002 groups; however, this increase was higher in the LY294002 group than in the H89 group (Fig. 2A). With high iodine concentrations, the expression of NIS was significantly decreased in the H89 group, whereas the expression of NIS was still increased in the LY294002 group (Fig. 2B). These results indicate that the TSH-mediated cAMP-PKA and PLC-IP3 pathways have different effects on NIS expression, due to the different intracellular iodinated compounds that are formed by treating cells with different iodine concentrations.

\section{Discussion}

Much of the data regarding the effect of iodine on NIS expression and activity is the result of research on the mechanisms of the Wolff-Chaikoff effect. Evidence that an acute high dose of iodine blocks iodide organification by inhibiting the activity of TPO and reduces the expression of NIS at both the mRNA and protein levels has been presented $(16,21)$. A previous study shows that methimazole, an inhibitor of TPO activity, blocks iodine organification and abolishes an acute Wolff-Chaikoff effect (15). These findings reveal that intracellular or follicular iodinated compounds may be responsible for the regulation of NIS expression and iodine uptake. However, results from studies carried out in tissue or animal models cannot exclude the interference of follicular organic iodinated compounds, such as iodinated $\mathrm{Tg}$, which exists within intact thyroid follicle lumen.

In this study, we investigated the effect of intracellular iodinated compounds on the regulation of NIS expression in a pig monolayer thyrocyte in vitro, and found that thyroid follicle lumen failed to form and was not capable of storing organic iodinated compounds. High iodine concentrations increased NIS expression at the mRNA and protein levels. This phenomenon was not observed with the addition of MMI (Fig. 1A and B). These effects on regulation were similar on the 2nd, 3rd and 4th day after the monolayer cells were treated with drugs (Fig. 1C). Our findings indicate that intracellular iodinated compounds are responsible for the increase in NIS expression.

We then investigated the effect of the TSH-mediated CAMP-PKA and PLC-IP3 pathways on the expression of NIS by inhibiting the activity of PAK or IP3 in the presence of different iodine concentrations. Both the cAMP-PKA and PLC-IP3 signaling pathways inhibited the expression of NIS with low concentrations of iodine, though the effect of the PLC-IP3 pathway was more marked than that of the cAMP-PKA pathway. The inhibition of IP3 activity by LY294002 resulted in higher NIS expression than the suppression of PKA activity by H89 (Fig. 2A).

Of note, the effect of the cAMP-PKA pathway on NIS expression changed from inhibition to promotion with high concentrations of iodine. The expression of NIS was significantly decreased rather than increased compared to the control group when APK activity was inhibited by H89 (Fig. 2B).

We showed that intracellular iodinated compounds promote NIS expression by altering the effects of the TSH-mediated cAMP-PKA and PLC-IP3 pathways, and that high intracellular iodinated compounds reversed the effect of the TSH-mediated 
cAMP-PKA pathway on the expression of NIS, from inhibition to promotion. This finding may explain the observed up-regulation of NIS when a high dose of iodine was administrated. It also strongly supports the notion that intracellular iodinated compounds disturb TSH-mediated adenylate cyclase activity, as indicated by recent studies $(16,22)$.

The results of the present study contradict the reported results obtained using tissue and animal models possessing thyroid follicles. This discrepancy may be attributed to follicular $\mathrm{Tg}$, which has been confirmed to inhibit thyroid-specific genes and iodine uptake activity (23), and to regulate follicular function through negative feedback (24).

The incidence of thyroid dysfunction and autoimmune thyroid diseases caused by excessive iodine has significantly increased $(25,26)$. The present findings may shed the light on the pathogenesis of these thyroid diseases.

\section{Acknowledgements}

We are grateful to Guangjun Jing for valuable advice and excellent technical assistance. This study was supported by the Natural Science Foundation of Fujian Province (grant no. C0510013) and the Professor's fund.

\section{References}

1. Dai G, Levy O and Carrasco N: Cloning and characterization of the thyroid iodide transporter. Nature 379: 458-460, 1996.

2. Dwyer RM, Bergert ER, O'Connor MK, Gendler SJ and Morris JC: Sodium iodide symporter-mediated radioiodide imaging and therapy of ovarian tumor xenografts in mice. Gene Ther 13: 60-66, 2006

3. Dohan O, De la Vieja A, Paroder V, et al: The sodium/iodide symporter (NIS): characterization, regulation, and medical significance. Endocr Rev 24: 48-77, 2003.

4. De la Vieja A, Dohan O, Levy O and Carrasco N: Molecular analysis of the sodium/iodide symporter: impact on thyroid and extrathyroid pathophysiology. Physiol Rev 80: 1083-1105, 2000.

5. Vassart G and Dumont JE: The thyrotropin receptor and the regulation of thyrocyte function and growth. Endocr Rev 13: 596-611, 1992.

6. Riedel C, Levy O and Carrasco N: Post-transcriptional regulation of the sodium/iodide symporter by thyrotropin. J Biol Chem 276: 21458-21463, 2001

7. Paire A, Bernier-Valentin F, Selmi-Ruby S and Rousset B: Characterization of the rat thyroid iodide transporter using anti-peptide antibodies. Relationship between its expression and activity. J Biol Chem 272: 18245-18249, 1997.

8. Levy O, Dai G, Riedel C, et al: Characterization of the thyroid $\mathrm{Na}+/ \mathrm{I}-$ symporter with an anti-COOH terminus antibody. Proc Natl Acad Sci USA 94: 5568-5573, 1997.

9. Saito T, Endo T, Kawaguchi A, et al: Increased expression of the Nat/I- symporter in cultured human thyroid cells exposed to thyrotropin and in Graves' thyroid tissue. J Clin Endocrinol Metab 82: 3331-3336, 1997.
10. Kogai T, Curcio F, Hyman S, Cornford EM, Brent GA and Hershman JM: Induction of follicle formation in long-term cultured normal human thyroid cells treated with thyrotropin stimulates iodide uptake but not sodium/iodide symporter messenger RNA and protein expression. J Endocrinol 167: 125-135, 2000.

11. Weiss SJ, Philp NJ, Ambesi-Impiombato FS and Grollman EF: Thyrotropin-stimulated iodide transport mediated by adenosine 3',5'-monophosphate and dependent on protein synthesis. Endocrinology 114: 1099-1107, 1984.

12. Eng PH, Cardona GR, Fang SL, et al: Escape from the acute Wolff-Chaikoff effect is associated with a decrease in thyroid sodium/iodide symporter messenger ribonucleic acid and protein. Endocrinology 140: 3404-3410, 1999.

13. Wolff J, Chaikoff IL, Goldberg RC and Meier JR: The temporary nature of the inhibitory action of excess iodine on organic iodine synthesis in the normal thyroid. Endocrinology 45: 504-513, 1949.

14. Schmutzler C: Regulation of the sodium/iodide symporter by retinoids - a review. Exp Clin Endocrinol Diabetes 109: 41-44, 2001.

15. Ferreira AC, Lima LP, Araujo RL, et al: Rapid regulation of thyroid sodium-iodide symporter activity by thyrotrophin and iodine. J Endocrinol 184: 69-76, 2005.

16. Serrano-Nascimento C, Calil-Silveira $J$ and Nunes MT: Posttranscriptional regulation of sodium-iodide symporter mRNA expression in the rat thyroid gland by acute iodide administration. Am J Physiol Cell Physiol 298: C893-C899, 2010.

17. Grollman EF, Smolar A, Ommaya A, Tombaccini D and Santisteban P: Iodine suppression of iodide uptake in FRTL-5 thyroid cells. Endocrinology 118: 2477-2482, 1986.

18. Kleinau G, Haas AK, Neumann S, et al: Signaling-sensitive amino acids surround the allosteric ligand binding site of the thyrotropin receptor. FASEB J 24: 2347-2354, 2010.

19. Trinquet E, Fink M, Bazin H, et al: D-myo-inositol 1-phosphate as a surrogate of D-myo-inositol 1,4,5-tris phosphate to monitor $\mathrm{G}$ protein-coupled receptor activation. Anal Biochem 358: 126-135, 2006.

20. Dudkin EA, Sheffield JB and Gruberg ER: Combining visual information from the two eyes: the relationship between isthmotectal cells that project to ipsilateral and to contralateral optic tectum using fluorescent retrograde labels in the frog, Rana pipiens. J Comp Neurol 502: 38-54, 2007.

21. Leoni SG, Galante PA, Ricarte-Filho JC and Kimura ET: Differential gene expression analysis of iodide-treated rat thyroid follicular cell line PCCl3. Genomics 91: 356-366, 2008.

22. Thomasz L, Oglio R, Rivandeira DT, et al: Inhibition of goiter growth and of cyclic AMP formation in rat thyroid by 2-iodohexadecanal. Mol Cell Endocrinol 317: 141-147, 2010.

23. Ulianich L, Suzuki K, Mori A, et al: Follicular thyroglobulin (TG) suppression of thyroid-restricted genes involves the apical membrane asialoglycoprotein receptor and TG phosphorylation. J Biol Chem 274: 25099-25107, 1999.

24. Suzuki K, Mori A, Saito J, Moriyama E, Ullianich L and Kohn LD: Follicular thyroglobulin suppresses iodide uptake by suppressing expression of the sodium/iodide symporter gene. Endocrinology 140: 5422-5430, 1999.

25. Teng W, Shan Z, Teng X, et al: Effect of iodine intake on thyroid diseases in China. N Engl J Med 354: 2783-2793, 2006.

26. Laurberg P, Bulow Pedersen I, Knudsen N, Ovesen L and Andersen S: Environmental iodine intake affects the type of nonmalignant thyroid disease. Thyroid 11: 457-469, 2001. 